

\title{
Caracterización preliminar de la ceniza de cáscara de arroz de la provincia Manabí, Ecuador, para su empleo en hormigones
}

\author{
César M. Jarre Castro ${ }^{*}$ (D), René Antonio Puig Martínez ${ }^{2}$ (D) Camilo Zamora-Ledezma $^{3}$ (D), \\ Ezequiel Zamora-Ledezma ${ }^{4}$ (D) \\ ${ }^{1}$ Laboratorio de Hormigones, Universidad Técnica de Manabí (UTM), Ave. Urbina y Che Guevara, Portoviejo, \\ Manabí, Ecuador, CP. \\ ${ }^{2}$ Centro de Estudios de la Construcción y Arquitectura Tropical (CECAT), Facultad de Ingeniería Civil, \\ Universidad Tecnológica de La Habana “José Antonio Echeverría” (CUJAE), Ave. 114 No. 11901, Marianao \\ 19390, La Habana, Cuba. \\ ${ }^{3}$ School of Physical Sciences and Nanotechnology, Yachay Tech University, San Miguel de Urcuquí, Hacienda San \\ José s/n, Project Yachay, 100119, Ecuador. \\ ${ }^{4}$ Facultad de Ingeniería Agrícola, Universidad Técnica de Manabí (UTM), Av. Urbina y Che Guevara, Portoviejo, \\ Manabí, Ecuador. \\ *Autor de correspondencia: mjarre@utm.edu.ec \\ https://doi.org/10.22209/rt.v44n1a06 \\ Recepción: 11 de agosto de 2020 | Aceptación: 21 de octubre de 2020 | Publicación: 01 de enero de 2021
}

\section{Resumen}

Este artículo se describen las potencialidades de la ceniza proveniente de la quema controlada de la cáscara de arroz en la provincia de Manabí (Ecuador), como sustituto del cemento Portland empleado en hormigones, incluyendo la elección y preparación de muestras representativas de cáscara de arroz, caracterización cuantitativa y cualitativa de la cáscara, procedimiento de quema y caracterización de la ceniza resultante. Se evalúan los procedimientos empleados en la caracterización de la cáscara de arroz, demostrando la pertinencia de utilizar el ensayo de absorción nuclear, para determinar el porcentaje en peso de sílice en la masa de la muestra, y se caracteriza la ceniza obtenida de la quema a diferentes temperaturas y tiempos, mediante procedimientos de fluorescencia y difracción de rayos X.

Palabras clave: ceniza decáscara de arroz; sílice amorfa; hormigón; puzolana.

\section{Preliminary characterization of the rice husk ash from the Manabí province for its use in concrete}

\begin{abstract}
This article describes the potentialities of ash from controlled burning of rice husks in the province of Manabi (Ecuador), as a substitute for portland cement used in concrete, including selection and preparation of representative samples of rice husk, quantitative and qualitative characterization of the husk, burning procedure and characterization of the resulting ash. The procedures used in the characterization of the rice husk are evaluated, demonstrating the relevance of using the nuclear absorption test to determine the percentage by weight of silica in the mass of the sample and the ash obtained from the burns at different temperatures and times, using fluorescence and X-ray diffraction procedures.
\end{abstract}

Keywords: rice husk ash; amorphous silica; concrete; pozzolan. 


\section{Introducción}

Las puzolanas son materiales que contienen sílice activa que en sí mismas tienen poca o ninguna cualidad aglomerante, pero que mezcladas con cal en presencia de agua, fraguan y endurecen como cemento Portland. De manera general, las puzolanas se pueden dividir en dos grandes grupos: naturales, como las cenizas volcánicas y zeolitas; y artificiales, como las arcillas calcinadas, las cenizas pulverizadas de carbón de piedra y las cenizas provenientes de la quema de residuos agrícolas [1].

Los griegos, 400 a.C., fueron los primeros que emplearon puzolanas en morteros de cal. Más tarde los romanos no solo usaron piezas de cerámica, ladrillos y tejas pulverizadas para formar puzolanas artificiales, sino también descubrieron que algunos suelos volcánicos mezclados con cal eran excelentes para producir morteros hidráulicos. Esta experiencia romana continuó aplicándose con diferentes alternativas y en la actualidad, constituye una práctica internacional la utilización de puzolanas en la producción de cementos y hormigones [2]. Existe una continuidad en el estudio sobre el empleo de puzolanas, de origen natural o artificial, como sustituto parcial del cemento Portland en la producción de hormigones. Bonavetti et al. [3] destacan esta experiencia en la producción de hormigones autocompactantes con elevados desempeños y durabilidad, con un bajo contenido de cemento Portland producto de la adición de zeolita natural a modo de puzolana.

Una puzolana investigada como sustituto parcial del cemento Portland en la producción de morteros y hormigones, es la ceniza proveniente de la quema controlada de la cáscara de arroz. Yanguatin et al.[1] detallan que las combinaciones experimentadas de cenizas de cáscara de arroz con cemento Portland, permiten sustituir hasta un 30\% del cemento por cenizas, sin que se produzcan afectaciones en la resistencia a la compresión. Se menciona además, que el porcentaje óptimo de sustitución fluctúa alrededor del $20 \%$, con lo cual se logra un incremento cercano a $20 \%$ de la resistencia a la compresión, mejoría en la estabilidad química del hormigón e incremento de su durabilidad. Como efecto negativo se aprecia un incremento en la demanda de agua de amasado.

En Ecuador, por su parte, no hay muchos referentes sobre el empleo de la ceniza de cáscara de arroz en la producción de morteros y hormigones. En este país, la cáscara de arroz es un residuo agroindustrial de difícil deposición final, que en estos momentos tiene pocas aplicaciones, convirtiéndose en un subproducto voluminoso y contaminante. Es un material rico en sílice $[1,3,9]$, que ha llamado la atención en la industria de la construcción, como sustituto parcial del cemento Portland empleado en la producción de hormigones, pero todavía sin resultados concluyentes. Es por ello que, en el presente trabajo, se realizó una caracterización preliminar de las cenizas de la cáscara de arroz en vías de su posible utilización en la producción de hormigones, mediante las siguientes premisas:
Primero, caracterización de la cáscara de arroz de la provincia de Manabí, en particular la determinación de su contenido de sílice, por métodos novedosos de ensayo para lograr mayor exactitud, comparando con los estándares internacionales y valorando su viabilidad para ser usada para la producción de ceniza como sustituto parcial del cemento Portland en la producción de hormigones.

Segundo, caracterización de la ceniza resultante de la quema a diferentes temperaturas y tiempos de quema, empleando técnicas de espectrometría y difracción de rayos $\mathrm{X}$.

\section{Materiales y métodos}

\section{Producción de arroz en Ecuador}

En términos sociales y productivos, el cultivo del arroz es el más importante del Ecuador, ocupando aproximadamente la tercera parte de la superficie de productos transitorios del país [4]. Considerando el comportamiento del cultivo no puede establecerse una tendencia que se defina como ascenso, pues en algunas áreas su producción lo mismo aumenta que disminuye, siendo una multivariedad de factores los causantes de las fluctuaciones. Las provincias con mayor participación en la producción de arroz son Guayas, Los Ríos, Manabí, Loja y El Oro. Cronológicamente en cuanto a rendimiento, la media nacional durante el año 2015 fue de 5,24 t/ha, siendo Loja la provincia de mayor predominancia, con una media de 6,75 t/ha. La provincia que mostró menor rendimiento fue El Oro, con una producción media de 3,68 t/ha.

Ya en el año 2016 la encuesta de superficie y producción agropecuaria continua refleja en su estadística, que la superficie nacional sembrada en ese año fue de 385.039 miles de hectáreas, área verdaderamente significativa, de la cual fueron cosechadas 366.194 miles de hectáreas, obteniéndose una producción de 1.534.537 t y ventas de 1.432.318 t [5].

Aunque es difícil lograr una estadística global debido a las omisiones por consumo local, los datos señalan que las principales provincias productoras de arroz son Guayas, Los Ríos y Manabí, sobre todo la primera, debido a sus suelos y condiciones climáticas favorables [6]. No obstante, en Ecuador suelen registrarse aumentos en la producción de arroz, pero a la vez reducciones en las áreas dedicadas al cultivo, por ser estas dedicadas a nuevos cultivos o existir criterios de rotación, tal como puede apreciarse en la Tabla 1 . Al analizar estos datos, se observa que las tres provincias mencionadas anteriormente poseen un peso del $96,6 \%$, como tendencia del total de arroz entero apilado en el país. Puede apreciarse además, de acuerdo con los datos obtenidos del MAGAP [7], que la producción de arroz de la provincia de Manabí en el año 2017, fue la tercera más importante del país, alcanzando 48.604 t; aunque solo representa el $3,37 \%$ de la producción nacional, el volumen es próximo a la suma del resto de las provincias del país, exceptuando Guayas y Los Ríos. 
Tabla 1. Producciones provinciales significativas de arroz en Ecuador, 2012-2017 [7].

\begin{tabular}{ccccccc}
\hline \multicolumn{7}{c}{ Producciones provinciales significativas de arroz en Ecuador, $\mathbf{t}$} \\
\hline \multirow{2}{*}{ Provincias } & $\mathbf{2 0 1 2}$ & $\mathbf{2 0 1 3}$ & $\mathbf{2 0 1 4}$ & $\mathbf{2 0 1 5}$ & $\mathbf{2 0 1 6}$ & $\mathbf{2 0 1 7}$ \\
\cline { 2 - 7 } & 1.029 .783 & 1.060 .669 & 902.424 & 1.187 .135 & 1.035 .344 & 986.397 \\
Guayas & 444.330 & 359.569 & 410.850 & 383.106 & 421.483 & 356.687 \\
Los Ríos & 42.128 & 63.656 & 45.607 & 57.169 & 55.536 & 48.604 \\
Manabí & 49.294 & 32.151 & 21.073 & 25.383 & 22.174 & 49.179 \\
Otras & 1.565 .535 & 1.516 .045 & 1.379 .954 & 1.652 .793 & 1.534 .537 & 1.440 .865 \\
Total (t) & 371.170 & 396.770 & 354.136 & 375.117 & 366.194 & 286.189 \\
Superficie (ha) & 4,22 & 3,82 & 3,90 & 4,41 & 4,19 & 5,03 \\
Rendimiento (t/ha) & &
\end{tabular}

En la provincia de Manabí, según informe del Banco Central del Ecuador [8], puede apreciarse que de los quince cantones (Olmedo, 24 de Mayo, Santa Ana, Rocafuerte, Sucre, Portoviejo, Paján, El Carmen, Chone, Pichincha, Tosagua, Junín, Flavio Alfaro, Jipijapa y Jama), los ocho primeros tienen el peso fundamental en la producción de arroz, con el $94 \%$ del total. Se destaca que colindan Santa Ana, 24 de Mayo y Olmedo; y lo mismo ocurre con los cantones de Portoviejo, Rocafuerte y Sucre. Ello explica la localización de las principales piladoras que realizan el descascarado del arroz en cáscara, seco y limpio, de estos seis cantones, las cuales se encuentran en Santa Ana y Portoviejo. Precisamente el arroz de Rocafuerte y Sucre se trae en su mayoría a los centros procesadores de Portoviejo, y hacia Santa Ana el arroz de los cantones de 24 de Mayo y Olmedo. En el caso de Paján y El Carmen, el arroz cultivado se procesa en piladoras de los mismos cantones.

Este análisis anterior permitió a los efectos de esta investigación, sintetizar las cuatro regiones fundamentales de la provincia, detallándose producción porcentual media y localización de la principal piladora, con la intención de definir las actividades de muestreo de la cáscara de arroz a utilizar en los ensayos:

- Región 1: cantones de Santa Ana, 24 de Mayo y Olmedo; $31 \%$ de la producción provincial; principal piladora en Santa Ana.

- Región 2: cantones de Sucre, Rocafuerte y Portoviejo; $27 \%$ de la producción provincial; principal piladora en Portoviejo.

- Región 3: al sur, solo el cantón Paján, 21\% de la producción provincial; principal piladora en centro del propio cantón.

- Región 4: al norte, solo el cantón El Carmen; 16\% de la producción provincial; principal piladora en centro del propio cantón.

Para mejor comprensión sobre la distribución de la cáscara de arroz con fines de muestreo, en la Figura 1 se detallan espacialmente las cuatro regiones en la provincia de Manabí y la ubicación de las piladoras en ellas.

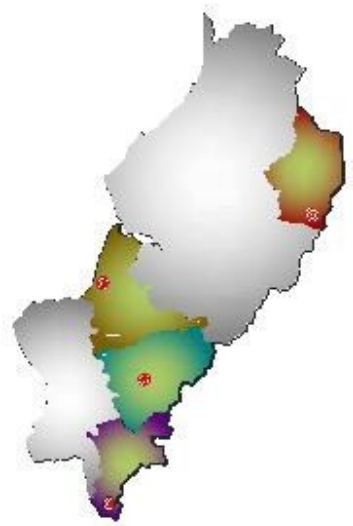

Simbología:

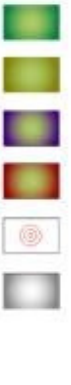

Región 1

Región 2

Región 3

Región 4

Area de piladoras

Resto de la provincia

Figura 1. Ubicación de las cuatro grandes regiones arroceras y de las piladoras en ellas [9].

También se constata en los informes especializados que dentro de cada una de las regiones sintetizadas prima una misma variedad de arroz a cultivar, lo que constituye una ventaja que facilita el muestreo posterior, dirigido a la caracterización física y química de la cáscara de arroz. El dato más relevante es la estimación media de los residuales productivos. Si la producción de arroz en la provincia se mantuviera o se incrementase, pueden estimarse no menos de 13,4 miles de toneladas anuales de cáscara de arroz en las regiones seleccionadas, que en vez de contaminar el ambiente y con una política bien dirigida, pueden ser empleadas como materia prima para la producción de hormigones, como sustituto parcial del cemento Portland, justificando plenamente esta investigación y su aplicación industrial.

A los efectos de la presente investigación interesa la producción de arroz en cáscara seco y limpio, ya que este es el arroz que llega a las piladoras para el proceso de descascarado. Consultas efectuadas en la zona permitendefinir que producto del proceso de pilado, aproximadamente $20 \%$ del peso de arroz en cáscara seco 
y limpio constituye la cáscara, y considerando los altos volúmenes productivos en la zona, el potencial regional es alto [10].

\section{Contenido de sílice en la cáscara de arroz}

Considerando el análisis anterior sobre la distribución del cultivo y procesamiento de arroz en Ecuador, se seleccionaron muestras procedentes de piladoras de Paján, Santa Ana, San Eloy, Rocafuerte y Chone, garantizándose así la representatividad tanto del norte, como del centro y sur del país.

La presencia de sílice dentro de la estructura de la cáscara de arroz se conoce desde 1938, la cual oscila alrededor del 20\%, presentándose en mayor cantidad con respecto al grano de arroz $[9,10,11]$. Respecto al tipo de estructura, los polimorfos de sílice son varios: cuarzo, cristobalita, tridimita, coestita, stishovita, lechatelerita y gel de sílice. El sílice o dióxido de silicio $\left(\mathrm{SiO}_{2}\right)$, como también se conoce, generalmente existe en dos formas, amorfa y cristalina [11].

Para la determinación porcentual de la presencia de sílice en las muestras de cáscara de arroz de las cinco piladoras seleccionadas, se aplicó la técnica de absorción nuclear, una de las más novedosas y exactas a estos fines [12,13], enviando las mismas a los laboratorios de la Dirección de Ciencias Químicas y Ambientales de la Facultad de Ciencias Naturales y Matemáticas de la Escuela Politécnica del Litoral, Ecuador (ESPOL).

\section{Obtención de la ceniza de cáscara de arroz}

Concluida la caracterización de la cáscara de arroz, como siguiente paso en la investigación, se acometió el análisis del procedimiento de quema controlada y caracterización de la ceniza resultante.

La cáscara de arroz al ser sometida a un proceso de calcinación produce ceniza en el orden del 13 al 29\% del peso inicial, y está compuesta principalmente por sílice en proporción variable entre un 87 y 97\%, más otras cantidades de sales inorgánicas que pueden ser eliminadas.

Se ha documentado que en dependencia del rango de temperatura y la duración de la combustión, se obtienen formas cristalinas o amorfas de sílice [11,14]. En general, se acepta que la formación significativa de sílice amorfa se da en el rango de 600 a $800{ }^{\circ} \mathrm{C}$, mientras que la sílice cristalina se produce por encima $900{ }^{\circ} \mathrm{C}$ [14]. Las formas cristalinas y amorfas de la sílice tienen diferentes propiedades. Para la aplicación requerida en esta investigación, la relevancia es hacia cenizas con estructura amorfa, aspecto más proclive en temperaturas mínimas dentro del rango, aunque la formación de sílice es un poco inferior, siendo la relación de comportamiento inverso $[15,16]$.

Previo al proceso de calcinación, se realizó un proceso de secado colocando las muestras de cáscara patrones a temperatura controlada de $105{ }^{\circ} \mathrm{C}$ durante 24 horas. Tras lograr una humedad casi nula, el procedimiento de quema se efectuó en un horno SAMO Thermal (modelo LT3140), generándose en total 70 puntos operativos en el proceso de calcinación de la cáscara de arroz, tal como puede apreciarse en la Figura 2, donde las columnas verticales, de izquierda a derecha, corresponden a incrementos de temperatura desde 600 hasta $875^{\circ} \mathrm{C}$ con paso de $25^{\circ} \mathrm{C}$; verticalmente, de abajo hacia arriba, tiempos de quema entre 15 y $90 \mathrm{~min}$, con paso de $15 \mathrm{~min}$. Pueden apreciarse claramente los cambios de coloración en cada rango.

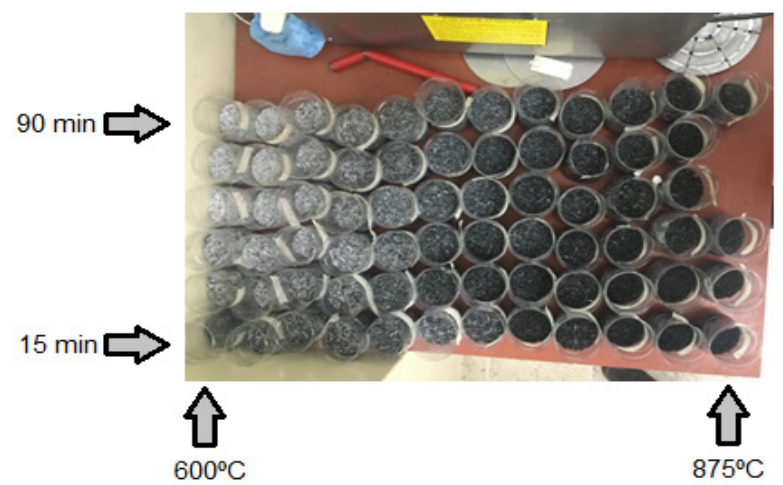

Figura 2. Puntos operativos obtenidos en el proceso de calcinación de cáscara de arroz.

Según resalta Salazar [17], como la ceniza de sílice es un bioproducto de un compuesto natural, en este caso la cáscara de arroz, requiere de cuidadosos métodos o procedimientos de estudios de caracterización, para comprender mejor su naturaleza y poder determinar las modificaciones necesarias en el proceso de obtención de sílice para mejorar sus propiedades, entre ellas la adherencia a la matriz, cuando se utiliza como relleno en los materiales compuestos.

\section{Caracterización de la ceniza de cáscara de arroz}

Las técnicas experimentales se basan en procedimientos estrictamente desarrollados para analizar la presencia y formación de sílice en ceniza de la cáscara de arroz, destacando entre ellas: la espectroscopía de resonancia magnética nuclear (RMN), microscopía electrónica de barrido (MEB), difracción de rayos de $X$ (DRX), fluorescencia de rayos $X$ (XRF), infrarrojo con transformada de Fourier (FTIR) y análisis térmico diferencial (ATD). En el caso de la presente investigación, para la estimación de la composición química de la ceniza de cáscara de arroz, fue empleada la técnica de $\mathrm{XRF}[18,19]$, en los laboratorios de química aplicada de la empresa de cementos CURAZAO SA, de la provincia de Artemisa, en Cuba; y para la caracterización estructural de la propia ceniza, la técnica de DRX [20,21,22], en los laboratorios de química de la Yachay Tech University, de San Miguel de Urcuquí, Ecuador.

\section{Resultados y discusión}

El ensayo de absorción nuclear a las muestras de cáscara de arroz de las piladoras seleccionadas, permite 
conocer su constitución porcentual de $\mathrm{SiO}_{2}$, tal como se recoge en la Tabla 2. Se aprecia que los resultados se ubican en el entorno de los rangos referenciados a nivel internacional [23]. Soares et al. [24] señalan un contenido medio de sílice entre 16 y $20 \%$ en la cáscara de arroz. Aunque dos de los valores encontrados resultan ligeramente inferiores en comparación a los restantes, los mismos no se consideran determinantes al no pertenecer a los ocho cantones productores del mayor volumen de cáscara generada, y dichos valores están solo un 1,7\% y un $1,0 \%$ de la cota inferior del $16 \%$ de presencia de sílice referenciado en la literatura. Resultados que evidencian la potencialidad del uso de la cascarilla indistintamente del cantón de origen de su procedencia.

Tabla 2. Análisis del porcentaje de sílice $\left(\mathrm{SiO}_{2}\right)$ en cáscara de arroz por la técnica de RMN.

\begin{tabular}{cc}
\hline Zona & $\%$ \\
\hline Paján & 15,0 \\
Santa Ana & 18,0 \\
San Eloy & 15,81 \\
Rocafuerte & 17,26 \\
Chone & 14,30 \\
\hline
\end{tabular}

Al evaluar inicialmente los resultados de la quema de la cáscara de arroz, se tuvo en cuenta la experiencia recogida en la literatura internacional. En este sentido, Ayhan [25] documenta que bajo una combustión completa, la ceniza de cáscara de arroz con mayor proporción de estructura amorfa, debe presentar una coloración que fluctúa en gris/blanco; pero que en condiciones de combustión parcial se produce una ceniza de color negro, más bien de estructura cristalina. Este resultado permite reducir a nueve los puntos operativos obtenidos producto de la quema controlada, seleccionando solo aquellos en que la coloración es gris/blanco, correspondientes a temperaturas de 600,625 y $650^{\circ} \mathrm{C}$, y tiempos de quema de 60, 75 y 90 min, mostrados en la Tabla 3. Estos fueron los empleados entonces para definir si la sílice en la ceniza de cáscara de arroz obtenida, poseía estructura amorfa o cristalina $[26,27]$.

Tabla 3. Muestras seleccionadas para la caracterización por ensayo de fluorescencia y difracción de rayos X.

\begin{tabular}{cccccccccc}
\hline \multirow{2}{*}{ Parámetros } & \multicolumn{10}{c}{ Muestras } \\
\cline { 2 - 10 } & M1 & M2 & M3 & M4 & M5 & M6 & M7 & M8 & M9 \\
\hline Tiempo [min] & 60 & 75 & 90 & 60 & 75 & 90 & 60 & 75 & 90 \\
Temperatura $\left[{ }^{\circ} \mathrm{C}\right]$ & 600 & 600 & 600 & 625 & 625 & 625 & 650 & 650 & 650 \\
\hline
\end{tabular}

Los resultados del análisis de FRX, para determinar la concentración porcentual de sílice en la ceniza, pueden ser apreciados en la Tabla 4. En todos los casos, se obtuvó un alto contenido de $\mathrm{SiO}_{2}$ en las muestras, con el valor máximo en la muestra M8, correspondiente a una temperatura de $650{ }^{\circ} \mathrm{C}$ y un tiempo de quema de 75 min.

Tabla 4. Resultados del porcentaje de sílice $\left(\mathrm{SiO}_{2}\right)$ y alúmina $\left(\mathrm{Al}_{2} \mathrm{O}_{3}\right)$ en las muestras de ceniza de cáscara de arroz.

\begin{tabular}{|c|c|c|c|c|c|c|c|c|c|}
\hline & \multicolumn{9}{|c|}{ Muestras } \\
\hline & M1 & M2 & M3 & M4 & M5 & M6 & M7 & M8 & M9 \\
\hline $\mathrm{SiO}_{2} / \mathrm{Si}$ & 85,00 & 88,85 & 87,21 & 88,76 & 88,59 & 89,31 & 90,05 & 90,62 & 90,26 \\
\hline \multicolumn{3}{|c|}{ Concentración media } & 88,74 & \multicolumn{4}{|c|}{ Concentración máxima } & 90,62 & \\
\hline \multicolumn{3}{|c|}{ Concentración mínima } & 85,00 & \multicolumn{4}{|c|}{ DST } & 1,74 & \\
\hline $\mathrm{Al}_{2} \mathrm{O}_{3} / \mathbf{A l}$ & 1,05 & 0,86 & 0,89 & 0,87 & 0,99 & 0,92 & 0,84 & 0,84 & 0,85 \\
\hline \multicolumn{3}{|c|}{ Concentración media } & 0,90 & \multicolumn{4}{|c|}{ Concentración máxima } & 1,05 & \\
\hline \multicolumn{3}{|c|}{ Concentración mínima } & 0,84 & \multicolumn{4}{|c|}{ DST } & 0,07 & \\
\hline
\end{tabular}

DST: : desviación estándar total

La caracterización mineralógica de las muestras de cenizas de cáscaras de arroz, efectuada mediante DRX, produjo difractogramas muy similares, prácticamente sin picos, tal como puede apreciarse en la Figura 3.

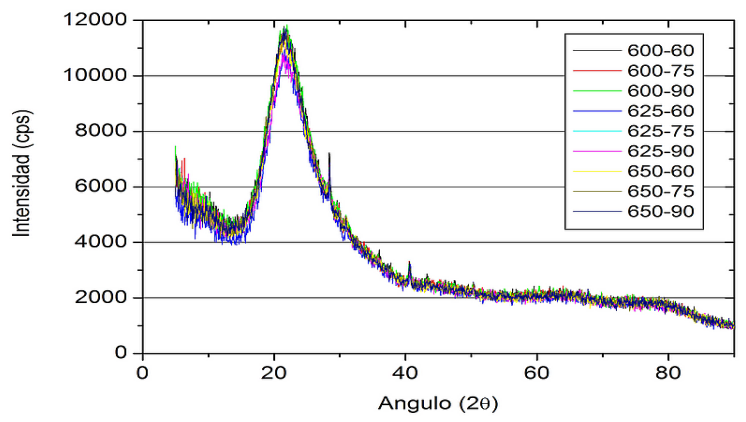

Figura 3. Difractogramas superpuestos de las muestras de cenizas de cáscaras de arroz, mediante difracción de rayos $\mathrm{X}$.

De acuerdo con lo reportado en la literatura especializada $[22,25,26]$, la abundancia de picos aguzados corresponden a una sílice cristalina o semicristalina, nombrada cristobalita. No obstante, del análisis de los difractogramas se puede apreciar que mineralógicamente, la estructura de las diferentes muestras experimentadas es predominantemente amorfa, lo que conviene a los intereses finales de la investigación.

Para finalizar, los resultados del presente trabajo permiten sentar las bases para dar continuidad al propósito final de la investigación, mediante la selección de muestras con una estructura mineralógica predominantemente amorfa, como la M6, tratada a una temperatura de 625 ${ }^{\circ} \mathrm{C}$ y un tiempo de quema de $90 \mathrm{~min}$, lo que constituye el resultado más prometedor de esta investigación. 


\section{Conclusiones}

La investigación realizada corrobora y demuestra que, el subproducto industrial de la producción de arroz en la provincia de Manabí, la ceniza de cáscara de arroz, cumple con los contenidos porcentuales de $\mathrm{SiO}_{2}$ que se reportan en la literatura internacional (entre 15 y 18\%), lo que fundamenta su posible uso en la fabricación de hormigones. En segundo lugar, la quema controlada de la misma, en rangos de temperatura entre 600 y 650 ${ }^{\circ} \mathrm{C}$, y tiempos entre 60 y 90 min, produce una ceniza que se caracteriza por una estructura mineralógica predominantemente amorfa, que avala su utilización como puzolana artificial sustitutiva del cemento Portland, en la elaboración de morteros y hormigones, y posibilita el diseño experimental de dosificaciones con diferentes niveles de sustitución de cemento por ceniza, recomendando para ello una temperatura de quema de $625^{\circ} \mathrm{C}$ y tiempo de quema de $90 \mathrm{~min}$.

\section{Referencias bibliográficas}

[1] Yanguatin H., Tobón J. y Ramírez J.: "Reactividad puzolánica de arcillas caoliníticas, una revisión". Revista Ingeniería de Construcción, Vol. 32, No. 2 (2017), 13-24.

[2] Jarre C. M. J., Albear J., Alcivar M. y Brito S.: "Impacto de la utilización de puzolanas naturales ecuatorianas". Revista de Investigaciones en Energía, Medio Ambiente y Tecnología (RIEMAT), Vol. 2, No. 1 (2017), $1-5$.

[3] Bonavetti V., Rahhal V., Locati F., Irassar E. F. Marfil, S. A. y Maiza, P.: "Utilización de una zeolita natural de la provincia de Chubut como adición mineral activa”. Memoria del VIII Congreso Internacional y $22^{a}$. Reunión Técnica de la Asociación Argentina de Tecnología del Hormigón, Olavarria, Argentina (2018).

[4] Ministerio de la Agricultura, Ganadería, Acuacultura y Pesca (MAGAP): "Boletín de Precios al Productor de MAGAP-Ecuador”. https://fliphtml5.com (2016), 17.

[5] Encuesta de Superficie y Producción Agropecuaria Continua (ESPAC): "Boletín de Estadísticas Agropecuarias, Instituto Nacional de Estadística y Censos de Ecuador (INEC)". https://www.ecuadorencifras.gob. ec (2016).

[6] Encuesta de Superficie y Producción Agropecuaria Continua (ESPAC): "Informe Ejecutivo. Instituto Nacional de Estadística y Censos de Ecuador (INEC)”. https://www. ecuadorencifras.gob.ec (2017).

[7] Ministerio de la Agricultura, Ganadería, Acuacultura y Pesca (MAGAP): "Boletín Situacional- Arroz". Published by Coordinación General del sistema de In- formación Nacional - CGSIN. https://fliphtml5.com (2017).

[8] Banco Central del Ecuador (BCE): "Reporte de Coyuntura del Sector Agropecuario-Banco Central del Ecuador", No. 90-II. https://contenido.bce.fin.ec (2017).

[9] Valverde-Arias O., Alberto J. \& Tarquis A.: "Using Geographical Information System to Generate a Drought Risk Map for Rice Cultivation: Case Study in Babahoyo Canton (Ecuador)". Biosystems Engineering Journal. Vol. 168, (2018), 26-41.

[10] Llanos O., Ríos A., Jaramillo C. A. y Rodríguez L.: "Rice husk as an alternative in decontamination processes". Revista Producción + Limpia, Vol. 11, No. 2 (2016), 150-160.

[11] Martínez J., Pineda T., López J. y Betancur M.: "Experimentos de combustión con cascarilla de arroz en lecho fluidizado para la producción de ceniza rica en sílice". Revista de la Facultad de Ingeniería de la Universidad de Antioquía, Vol. 51 (2010), 104-111.

[12] Vélez M., Murillo J., Rivadeneira A., Álava R. y Mendoza J.: "Evaluación de la cascara de arroz para fabricación de ladrillos". Pro Sciences jornal, Vol. 2, No. 11 (2018), 28-31.

[13] Bathla A., Narula C. \& Chauhan R. P.: "Hydrothermal synthesis and characterization of silica nanowires using rice husk ash: an agricultural waste". Journal of Materials Science: Materials in Electronics, Vol. 29, No. 8, (2018), 6225-6231.

[14] Chandrasekhar S., Pramada P. \& Majeed J.: "Effect of calcination temperature and heating rate on the optical properties and reactivity of rice husk ash". Revista Ciencia de los Materiales. Vol. 41, No. 23 (2006), 7926-7933.

[15] Xu W. T., Lo T. Y. \& Memon S. A.: "Microstructure and reactivity of rich husk ash". Revista Construcción y Materiales de Construcción. Vol. 29 (2012), 541-547.

[16] Zou Y. \& Yang T.: "Rice husk, Rice husk ash and their applications". In Rice Bran and Rice Bran Oil, AOCS Press (2019), 207-24.

[17] Salazar Carreño D., García Cáceres R. y Ortiz Rodríguez 0.: "Laboratory processing of Colombian rice husk for obtaining amorphous silica as concrete supplementary cementing material". Construction and Building Materials Journal, Vol. 96 (2015), 65-75.

[18] Mor S., Chhoden K. \& Ravindra K.: "Application of agro-waste rice husk ash for the removal of phosphate from the wastewater". Journal of Cleaner Production, No. 129 (2016), 673-680. 
[19] Bakar R. A., Yahya R. \& Gan S. N.: "Production of high purity amorphous silica from rice husk". Procedia Chemistry Journal, No. 19 (2016), 189-195.

[20] Wong Y. S., Kwan W. H. \& Lim M.: "Enhancing pozzolanic properties of rice husk ash using acid leaching treatment". In AIP Conference Proceedings, AIP Publishing LLC. September Vol. 2157, No. 1, (2019), 020027.

[21] Eliche Quesada D., Felipe Sesé M. A., López Pérez J. A. \& Infantes Molina A.: "Characterization and evaluation of rice husk ash and wood ash in sustainable clay matrix bricks". Ceramics International, Vol. 43, No. 1 (2017), 463-475.

[22] Viky-C Mujica F. \& Linares A.: "Effect of the synthesis method of $\mathrm{Ni}-\mathrm{Al}$ hydrocalcites as support of CoMo catalysts in hydrotreatment reactions". Revista Técnica de Ingeniería de Universidad del Zulia, Vol. 41, No. 1 (2018), 41-49.

[23] Fernandes I., Sánchez F., Jurado J., Kieling A., Rocha T., Moraes C. y Sousa V.: "Physical, chemical and electric characterization of thermally treated rice husk ash and its potential application as ceramic raw material". Advanced Powder Technology, Vol. 28, No. 4. (2017), 1228-1236.
[24] Soares L., Braga R., Freitas J., Ventura R., Pereira D. y Melo D.: "The effect of rice husk ash as pozzolan in addition to cement Portland class G for oil well cementing". Journal of Petroleum Science and Engineering, Vol. 131 (2015), 80-85.

[25] Ayhan D.: A discussion of the paper "Study on the pozzolanic properties of rice husk ash by hydrochloric acid pre-treatment" by Q. Feng, H. Yamamichi, M. Shoya, S. Sugita. Cement and Concrete Research, Vol. 35, (2005), 10-17.

[26] Wei Z., Wang Z., Tait W. R., Pokhrel M., Mao Y., Liu J., et al.: "Synthesis of green phosphors from highly active amorphous silica derived from rice husks". Journal of Materials Science, Vol. 53, No. 3 (2018), 1824-1832.

[27] Ananthi A., Geetha D. \& Ramesh P. S.: "Preparation and characterization of silica material from rice husk ash-an economically viable method". Chemistry and Materials Research, Vol. 8, No. 6 (2016), 1-7. 


\section{REVISTA TECNICA}

DE LA FACULTAD DE INGENIERIA

UNIVERSIDAD DEL ZULIA

Vol. 44. N`1, Enero - Abril 2021, pp. 04 - 58

Esta revista fue editada en formato digital y publicada en Diciembre de 2020, por el Fondo Editorial Serbiluz, Universidad del Zulia. Maracaibo-Venezuela

www.luz.edu.ve

www.serbi.luz.edu.ve

www.produccioncientificaluz.org 